\title{
Top quark pair production measurements using the ATLAS detector at the LHC
}

Sophie Trincaz-Duvoid (LPNHE-UPMC-IN2P3) on behalf of the ATLAS collaboration
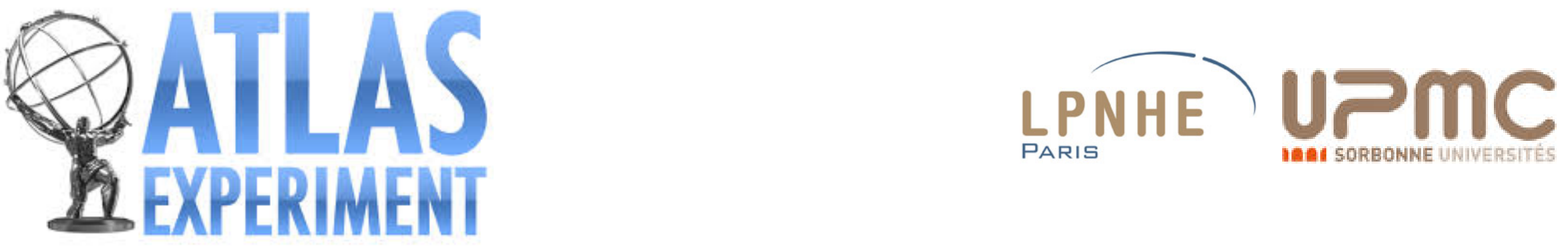


\section{Outline}

- Inclusive cross section

- Differential cross section measurements

- Production in association with gauge bosons 


\section{The interest of the Top Quark}

- Decay before hadronization -> access to bare quark

- The heaviest known fundamental particle $(173.34 \pm 0.76 \mathrm{GeV})$

$\Rightarrow$ special role in the electroweak Symmetry Breaking

- Sector to search for new physics

$\Rightarrow$ additional $\bar{t} \bar{t}$ production modes

$\Rightarrow$ additional $\bar{t} \bar{t}$ decay channels

$\Rightarrow$ background for many searches

- Tests/Information for QCD :

$\Rightarrow$ heavy quarks production calculation

$\Rightarrow$ test and tune MC models

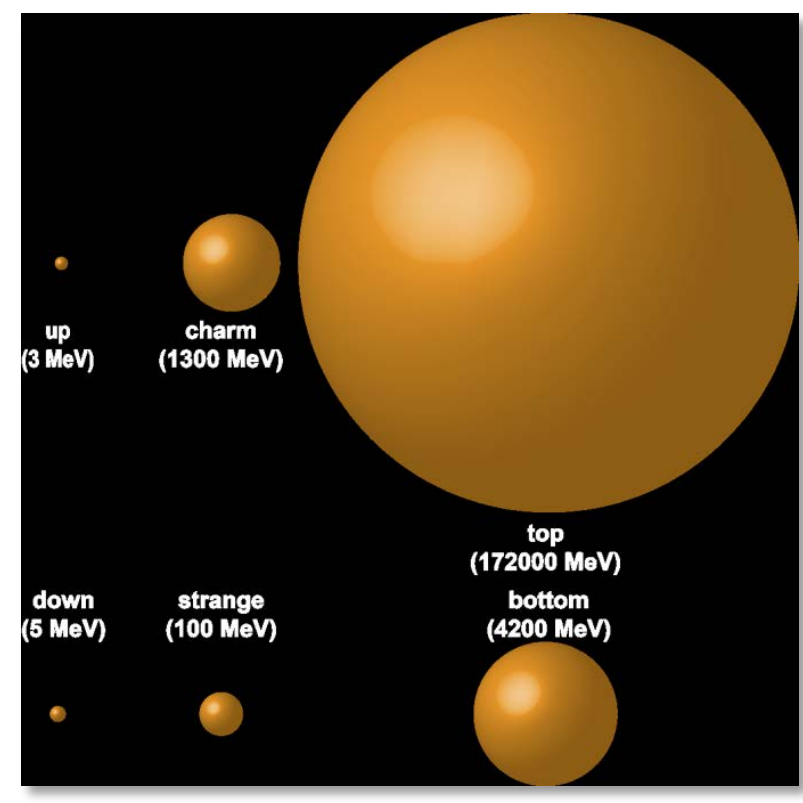

$\Rightarrow$ PDF - ISR/FSR Modeling 


\section{$\mathrm{t} \overline{\mathrm{t}}$ production and decay}

Top pair production at the LHC through gg (>84\%) and qq

Theory QCD precision NNLO + NNLL soft gluon resummation :
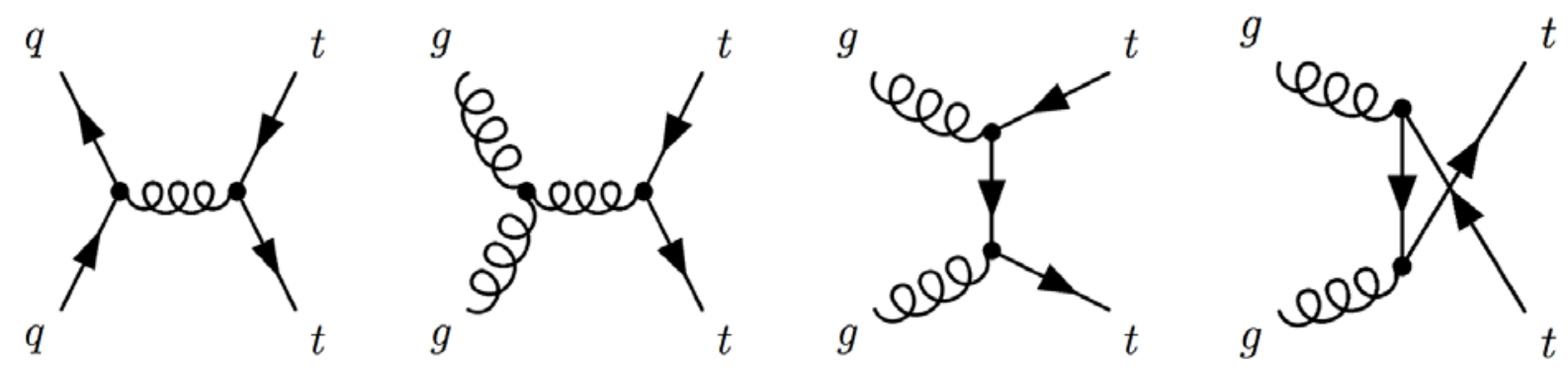

$\Rightarrow \sigma_{(\mathrm{pp} \rightarrow \mathrm{tt})}(7 \mathrm{TeV})=177_{-11}^{+10} \mathrm{pb}$

$\Rightarrow \sigma_{(\mathrm{pp} \rightarrow \mathrm{tt})}(8 \mathrm{TeV})=253_{-15}^{+13} \mathrm{pb}$

(at $\mathrm{m}_{\text {top }}=172.5 \mathrm{GeV}$ )

$\Rightarrow \sigma_{(\mathrm{pp} \rightarrow \mathrm{tt})}(13 \mathrm{TeV})=832_{-46}^{+40} \mathrm{pb}$

Czakon, Mitov, Fiedler, PRL 110 (2013) 252004

Top Pair Branching Fractions

- $t \rightarrow \mathrm{Wb}(\sim 100 \%)$

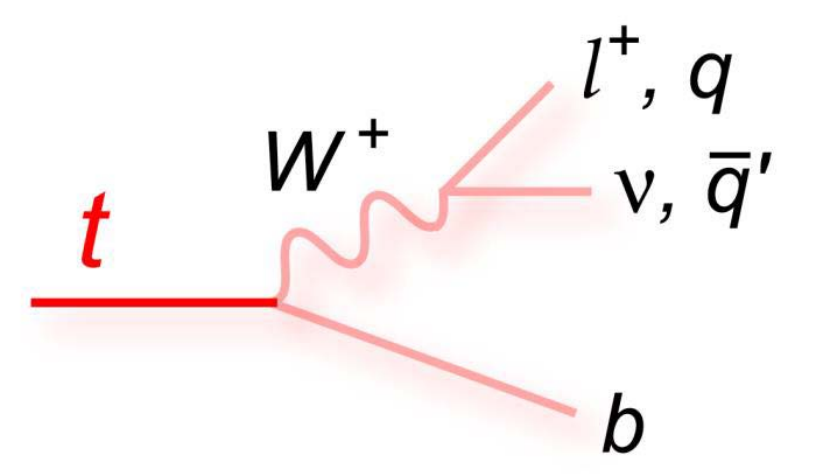

Final state depends on the $2 \mathrm{~W}$ decays

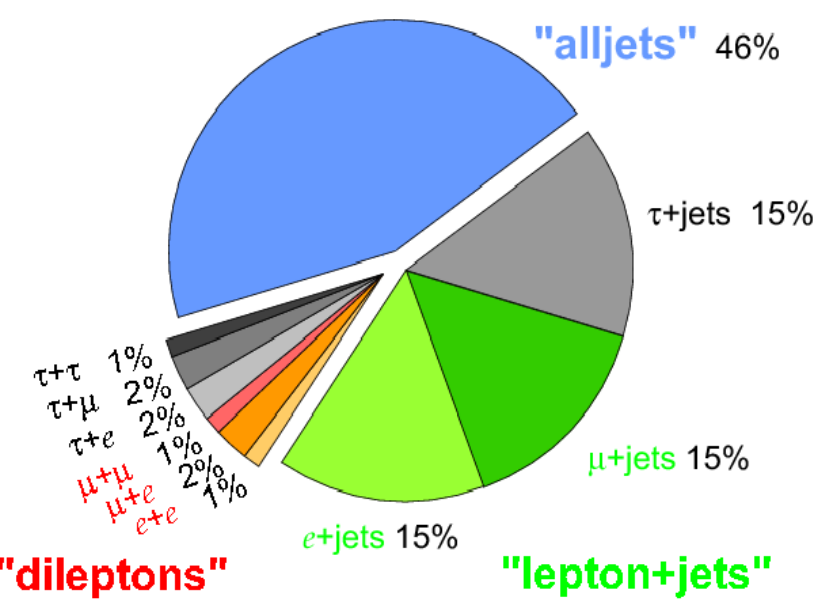




\section{Inclusive $t \mathrm{t}$ cross section in the}

\section{lepton+jets channel at $8 \mathrm{TeV}$}

\section{arXiv:1504.04251 - Submitted to JHEP}

- 1 e or $1 \mu+\geq 3$ jets (1b-tag)

$+E_{T}^{M i s s}$ and $M_{T}^{W}$

- Use a likelihood discriminant variable (LHD) built from lepton $\eta$ and transformed aplanarity $A^{\prime}$ $\mathrm{LHD}=\mathcal{L}^{\mathrm{s}} /\left(\mathcal{L}^{\mathrm{s}}+\mathcal{L}^{\mathrm{bkg}}\right)$

- Fit of template in binned LHD to data

- Syst dominated by MC modeling (including PDF, ISR/FSR)
ATLAS Data at $8 \mathrm{TeV}(2012): 20.3 \mathrm{fb}^{-1}$

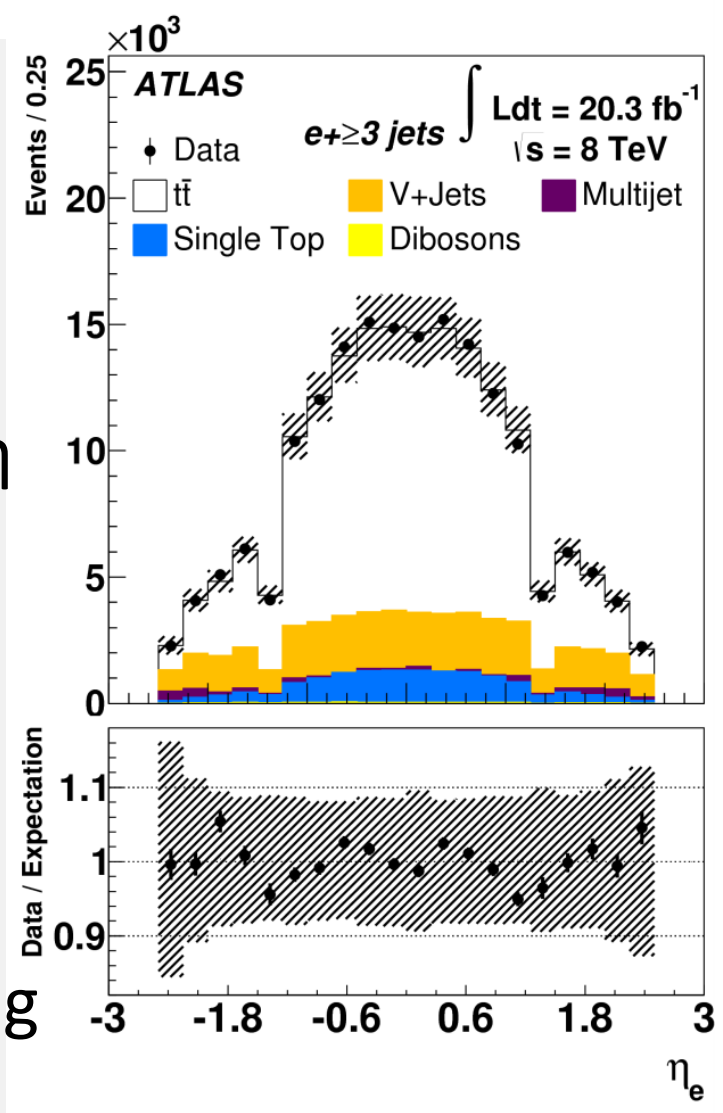

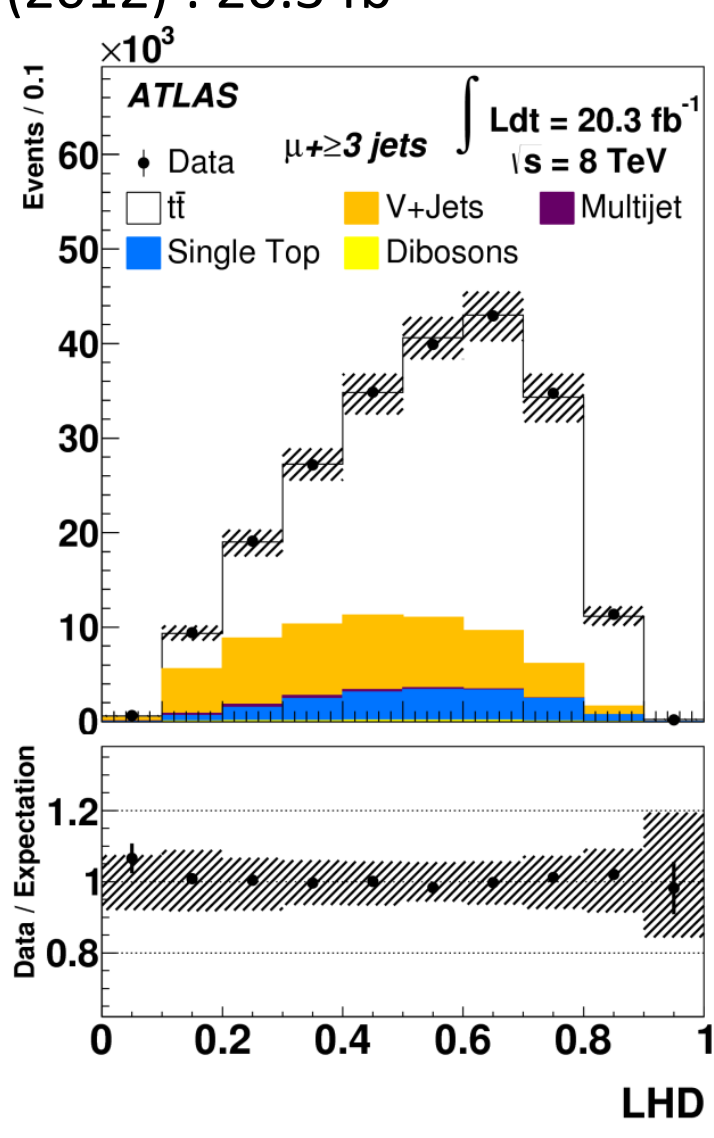

$\sigma(\mathrm{tt})=260 \pm 1$ (stat) $:-21$ (syst) \pm 8 (lumi) \pm 4 (beam) pb (8 TeV) (rel. error of $8 \%$ ) 


\section{Inclusive tt cross section - Summary}

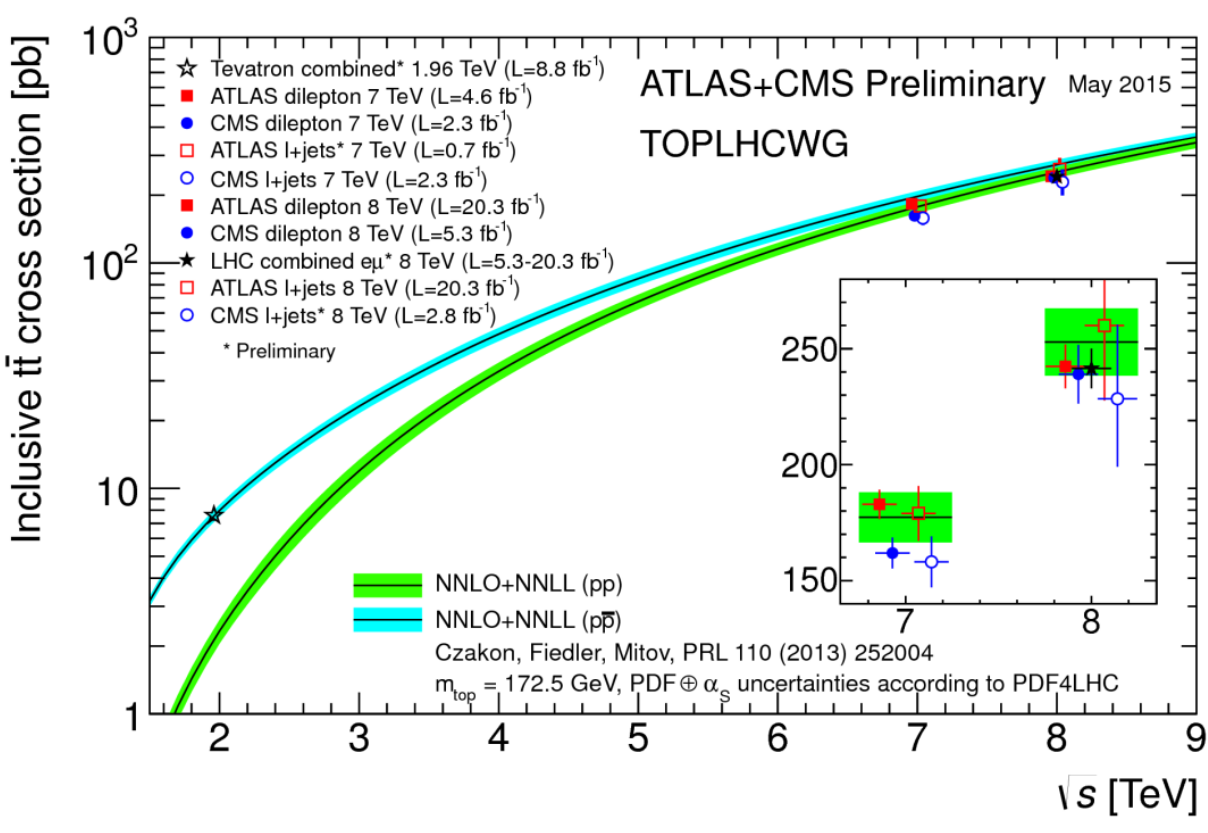

- Very precise QCD prediction to NNLO with NNLL corrections available since 2013

- All measurements are limited by systematics

- Best results from dilepton side - All measurements agree with predictions
ATLAS+CMS Preliminary $\sigma_{\mathrm{tf}}$ summary, is $=8 \mathrm{TeV}$ TOPLHCWG May 2015
NNLO+NNLL (Top ++2.0$)$, PDF4LHC $\mathrm{m}_{\text {top }}=172.5 \mathrm{GeV}$

scale uncertainty

scale $\oplus \mathrm{PDF} \oplus \alpha_{\mathrm{S}}$ uncertainty

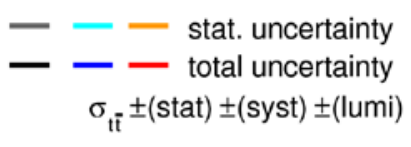

ATLAS, lepton+jets

arXiv: $1504.04251, L_{\text {int }}=20.3 \mathrm{fb}^{-1}$

$\begin{aligned} & \text { CMS prel., lepton+jets } \\ & \text { CMS-PAS TOP-12-006, } \mathrm{L}_{\text {int }}=2.8 \mathrm{fb}^{-1}\end{aligned}-228 \pm 9_{-26}^{+29} \pm 10 \mathrm{pb}$

CMS, lepton $+\tau_{\mathrm{h}}$

PLB 739 (2014) 23, $L_{\text {int }}=19.6 \mathrm{fb}^{-1}$

ATLAS, dilepton $\mathrm{e} \mu$

EPJ C74 (2014) 3109, $\mathrm{L}_{\text {int }}=20.3 \mathrm{fb}^{-1}$

CMS, dilepton (ee, $\mu \mu, \mathrm{e} \mu)$

JHEP 02 (2014) 024, $L_{\text {int }}=5.3 \mathrm{fb}^{-1}$

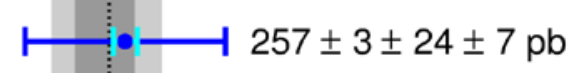

LHC combined e $\mu$ (Sep 2014)

CMS-PAS TOP-14-016,

ATLAS-CONF-2014-053,

$L_{\text {int }}=5.3-20.3 \mathrm{fb}^{-1}$

100

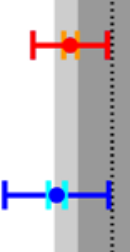

$242.4 \pm 1.7 \pm 5.5 \pm 7.5 \mathrm{pb}$

$239.0 \pm 2.1 \pm 11.3 \pm 6.2 \mathrm{pb}$

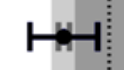

$241.5 \pm 1.4 \pm 5.7 \pm 6.2 \mathrm{pb}$

Effect of LHC beam energy uncertainty: $4.2 \mathrm{pb}$ (not included in the figure)

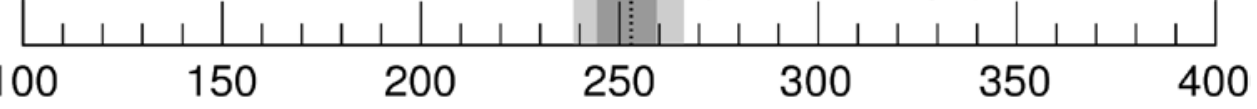

$$
\sigma_{\mathrm{tt}}[\mathrm{pb}]
$$




\section{Differential cross section}

Differential cross sections as a function of top or top pair variables:

$\Rightarrow \mathrm{P}_{\mathrm{T}}(\mathrm{t}), \mathrm{P}_{\mathrm{T}}(\mathrm{tt}), \mathrm{m}(\mathrm{tt}), \mathrm{y}(\mathrm{tt})$

Measurements in both fiducial (inside acceptance) and full phase space Probe perturbative QCD, test and tune MC models with data Top quark full kinematic reconstruction necessary and background subtraction Unfolding techniques used (correcting bin to bin migration):

$\Rightarrow$ Account for acceptance and detector effects (resolution, efficiency, etc.)

$\Rightarrow$ Correct observed distributions to parton-level (generated quarks) or particle-level (stable hadrons after fragmentation, parton shower ... ) Comparison with generators, PDF sets, calculations ... 
Differential cross section ( $\ell+j e t s)$ : from reco to parton-level

Phys. Rev. D 90, 072004

unfolded to top parton in full phase space Comparison with various $\mathrm{MC}$ generator with NLO-QCD and NLO+NNLL calculations Comparison with NLO predictions (MCFM) for different PDF sets

Main syst : JES, JER, I/FSR, PS, MC modeling
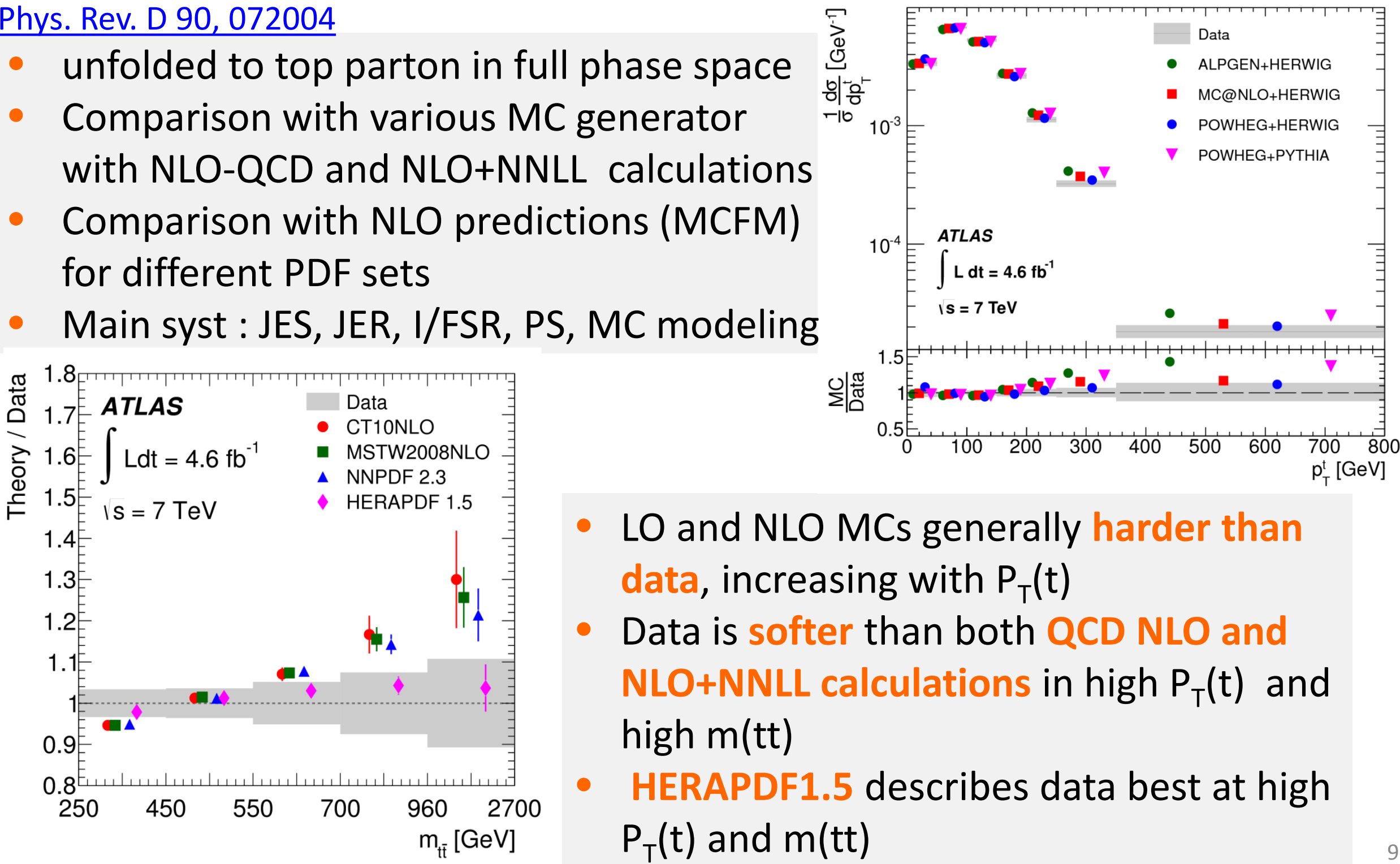

- LO and NLO MCs generally harder than data, increasing with $\mathrm{P}_{\mathrm{T}}(\mathrm{t})$

- Data is softer than both QCD NLO and $N L O+N N L L$ calculations in high $\mathrm{P}_{\mathrm{T}}(\mathrm{t})$ and high $\mathrm{m}(\mathrm{tt})$

- HERAPDF1.5 describes data best at high $\mathrm{P}_{\mathrm{T}}(\mathrm{t})$ and $\mathrm{m}(\mathrm{tt})$ 


\section{Differential cross section ( $\ell+j e t s)$ :}

\section{from reco to particle-level}

arXiv:1502.05923: Submitted

- Reconstruction of pseudo-top at detector level and particle level

$\Rightarrow$ less dependence on fragmentation, parton shower, hadronization, PDFs,... (often non-perturbative)

- Fiducial measurement (less model-dependant)

- Main systematics: b-tagging, jet energy, ISR/FSR, parton shower

- Similar results as at parton level but with lower uncertainties
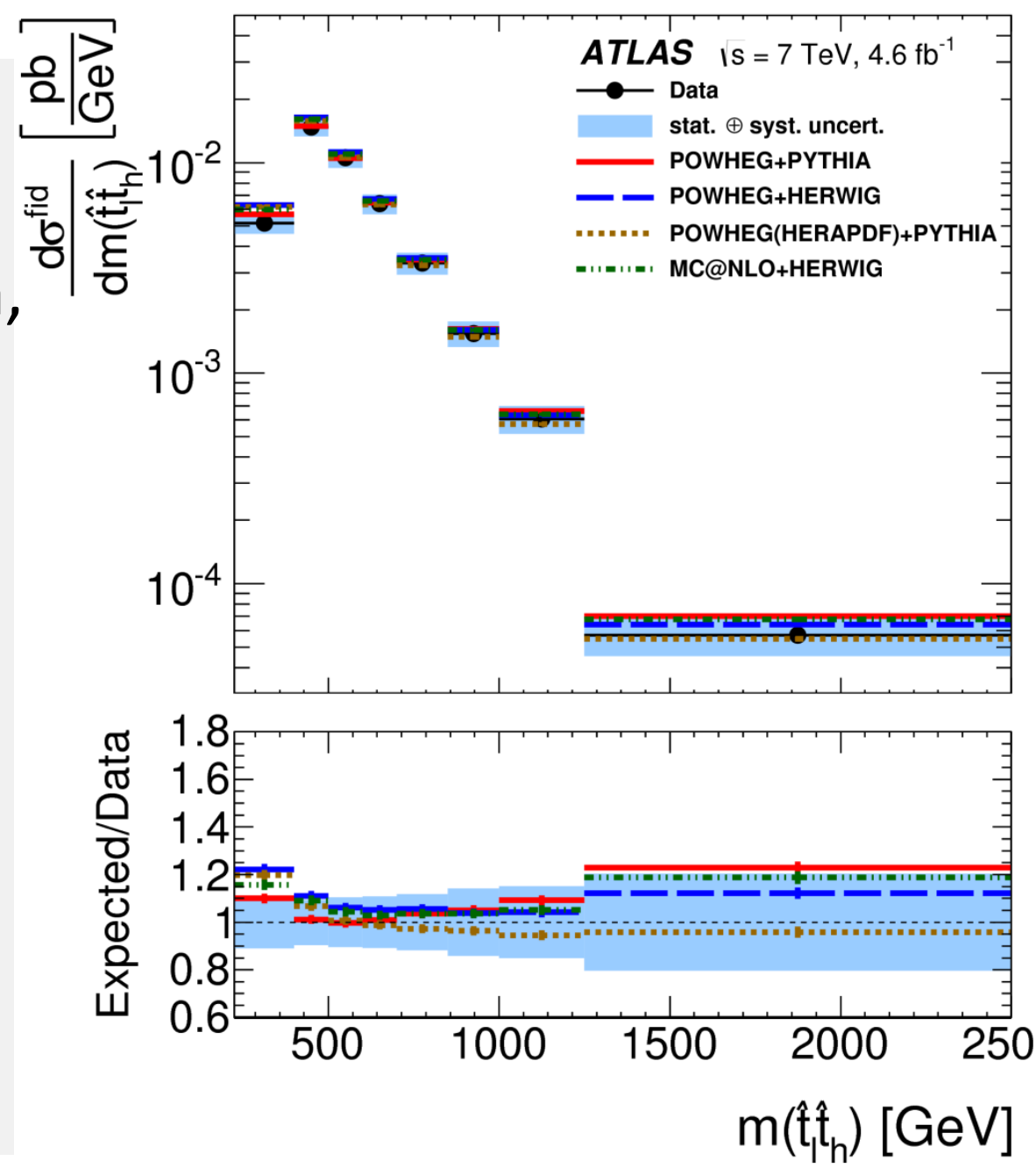



\section{Differential cross section for $t \bar{t}+j e t s$}

\section{JHEP01(2015)020}

$\sigma(t t+j e t s)$ as function of jet multiplicity or jets $\mathrm{P}_{\mathrm{T}}$ (several $\mathrm{P}_{\mathrm{T}}$ thresholds) at particle-level

- Sensitivity to parton shower modeling at high jet multiplicity

- Main systematics: from background modelling (at lower jet multiplicities) to jet energy scale (at higher jet multiplicities).

POWHEG+PYTHIA with limited hard radiations (hdamp): best Data description

- MC@NLO+HERWIG: predicts too few events at high jet multiplicities

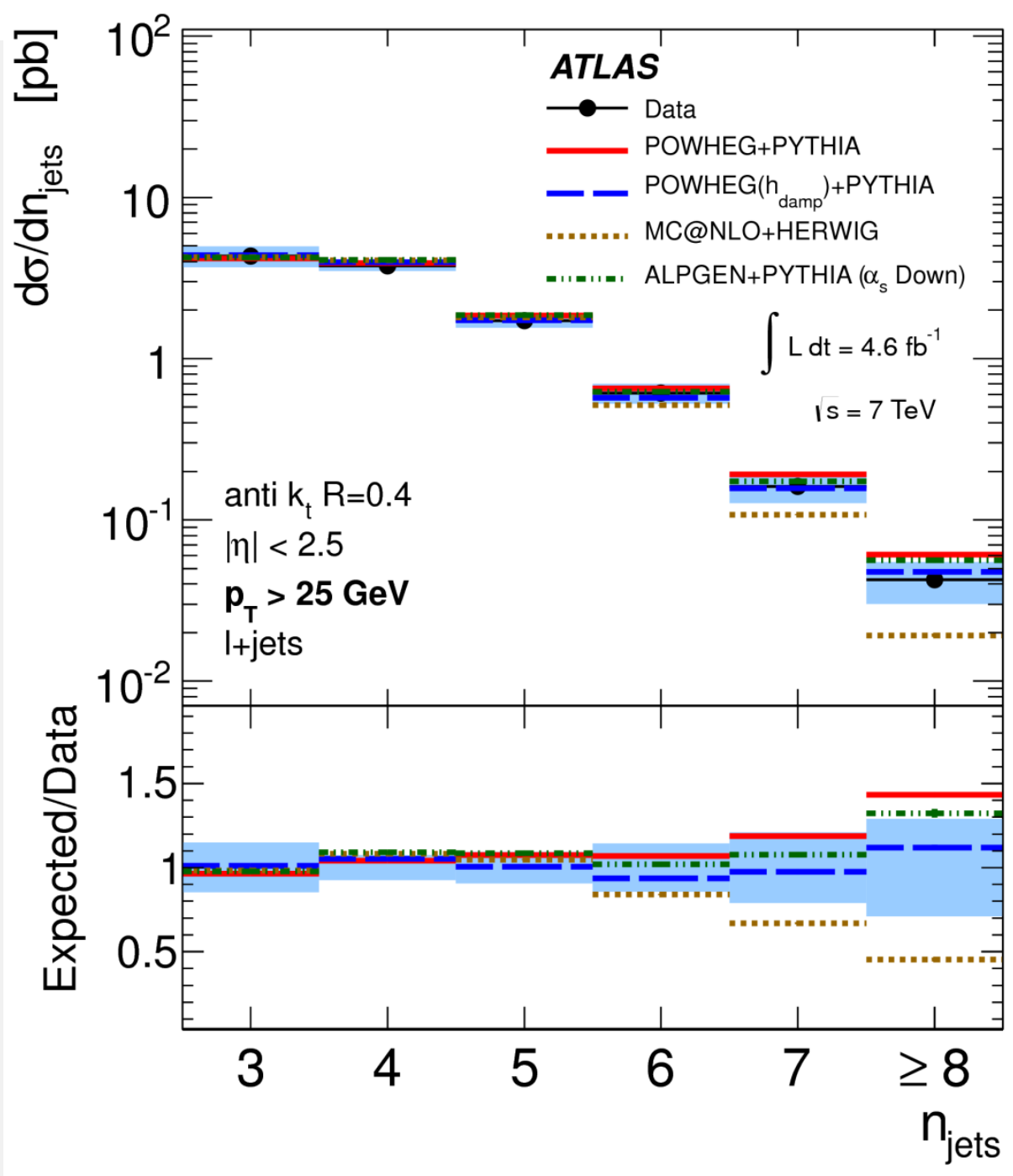




\section{$t \bar{t}+$ boson $(W, Z)$ measurement}

\section{ATLAS-CONF-2014-038}
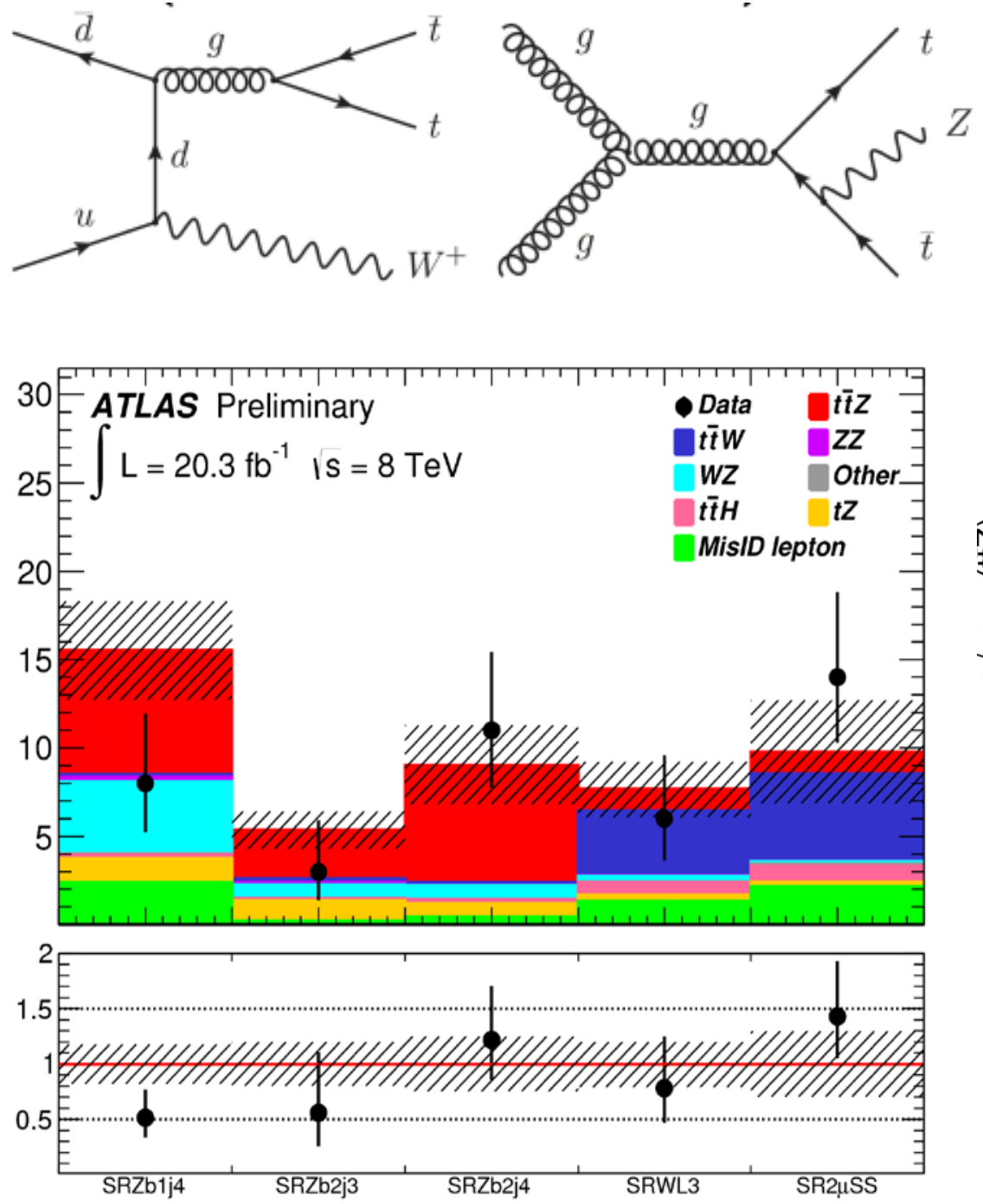

Sophie Trincaz-Duvoid - QCD2015
- dilepton (same sign and opposite sign)+ trilepton

- Background reduction by cuts on b-tags and jet multiplicity

- Dominated by statistics

- Evidence of ttZ and ttW: 3.10 (each)

- Cross sections consistent with NLO QCD calculations

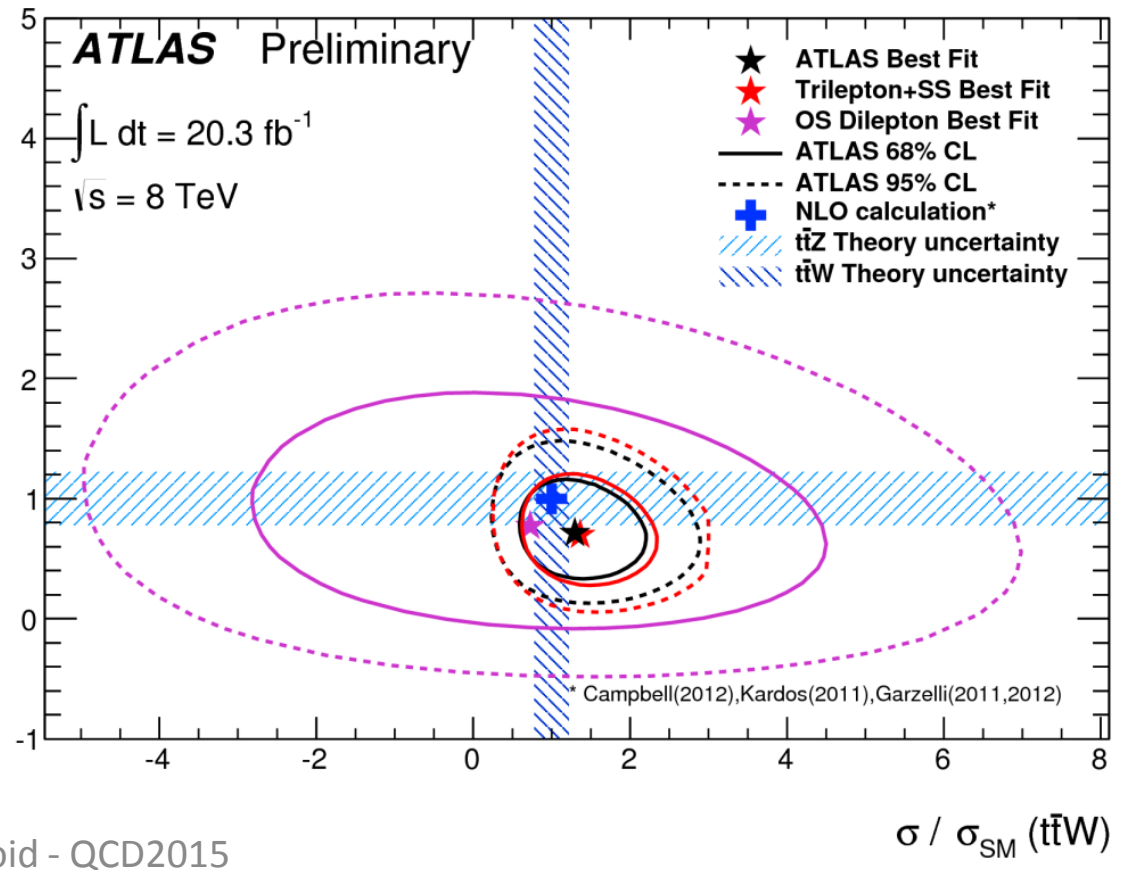




\section{Conclusion}

- Top quark pair production measurements in ATLAS have reached good precision level. Theoretical calculations challenged by precision of experimental data (at hadronic collider)

- Differential measurements (fiducial/inclusive) of tt production in top $\mathrm{P}_{\mathrm{T}}$ and other kinematics provide constraints to improve $\mathrm{MC}$ modeling and parameter tuning in tt production.

- Observation/evidence of associated production with gauge bosons. Cross section consistent with the SM.

- ATLAS is ready for more top physics in the TeV regime!

The ATLAS top program is very rich (see also next talk)

For more information and results https://twiki.cern.ch/twiki/bin/view/AtlasPublic/TopPublicResults 


\section{Extra Slides}




\section{Inclusive tt cross section in the dilepton channel $(\mathrm{e \mu})$ at 7 and $8 \mathrm{TeV}$}

Table 4. Summary of the relative statistical, systematic and total uncertainties on the measurements of the $t \bar{t}$ production cross-section $\sigma_{t \bar{t}}$ at $\sqrt{s}=7 \mathrm{TeV}$ and $\sqrt{s}=8 \mathrm{TeV}$.

\begin{tabular}{lcc}
\hline Uncertainty & \multicolumn{2}{c}{$\Delta \sigma_{t \bar{t}} / \sigma_{t \bar{t}}(\%)$} \\
$\sqrt{s}$ & $7 \mathrm{TeV}$ & $8 \mathrm{TeV}$ \\
\hline Data statistics & 1.69 & 0.71 \\
\hline$t \bar{t}$ modelling and QCD scale & 1.46 & 1.26 \\
Parton distribution functions & 1.04 & 1.13 \\
Background modelling & 0.83 & 0.83 \\
Lepton efficiencies & 0.87 & 0.88 \\
Jets and $b$-tagging & 0.58 & 0.82 \\
Misidentified leptons & 0.41 & 0.34 \\
\hline Analysis systematics $\left(\sigma_{t \bar{t}}\right)$ & 2.27 & 2.26 \\
\hline Integrated luminosity & 1.98 & 3.10 \\
LHC beam energy & 1.79 & 1.72 \\
\hline Total uncertainty & 3.89 & 4.27 \\
\hline
\end{tabular}

As shown in Tables 3 and 4, the largest systematic uncertainties on $\sigma_{t \bar{t}}$ come from $t \bar{t}$ modelling and PDFs, and knowledge of the integrated luminosities and LHC beam energy.
Correlation between $\sigma(\mathrm{tt})$ and $m_{t o p}^{\text {pole }}$

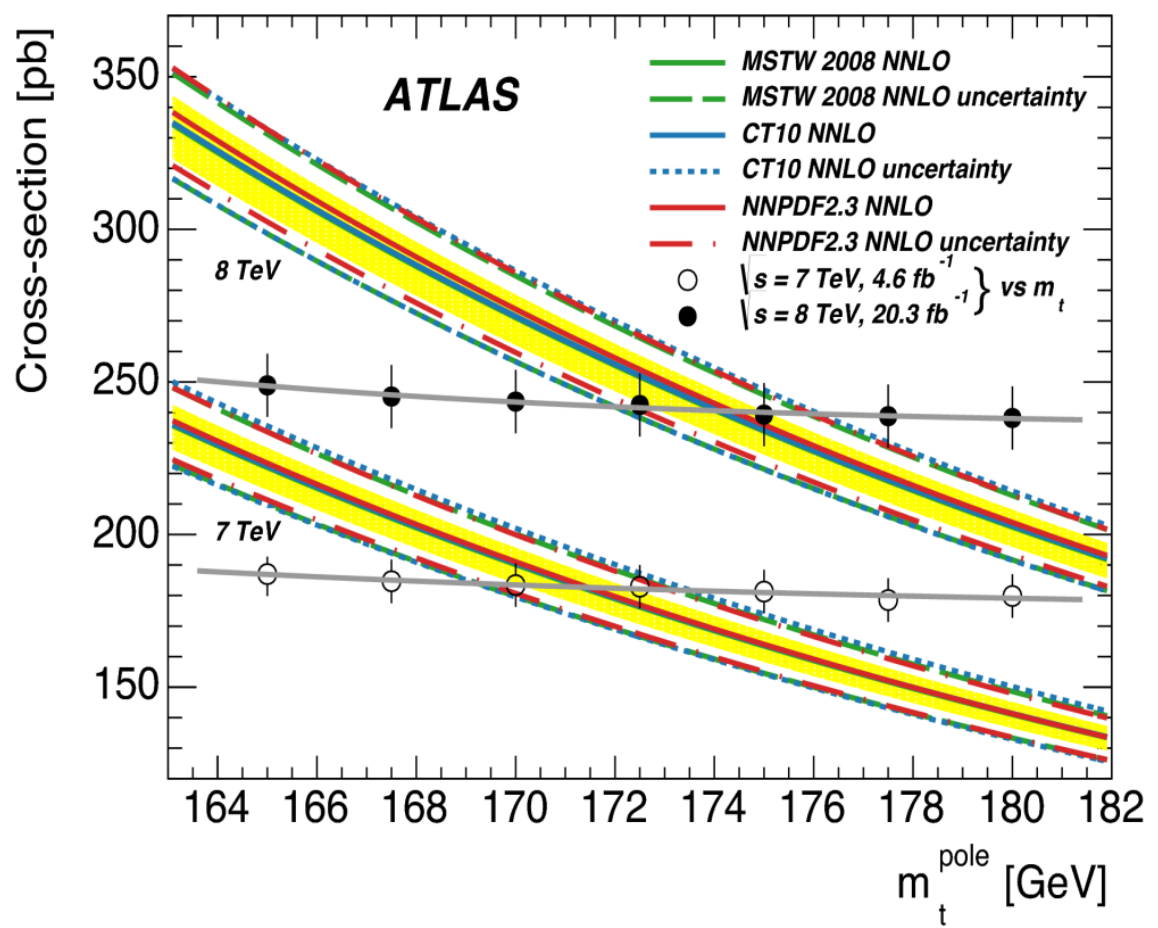

Combination 7 and $8 \mathrm{TeV}$ : $m_{\text {top }}^{\text {pole }}=172.9_{-2.6}^{+2.5} \mathrm{GeV}$ 


\section{Inclusive tt cross section in the lepton+jets channel at $8 \mathrm{TeV}$}

Table 2: Summary of the systematic uncertainties in the measurements of the $t \bar{t}$ production cross-section (\%). The bottom part of the table lists systematic uncertainties for the fiducial $t \bar{t}$ production cross-section for the cases where they are different from those for the inclusive one.

\begin{tabular}{lccc} 
Uncertainty on inclusive $\sigma_{t \bar{t}}$ & $e+$ jets & $\mu+$ jets & $\ell+$ jets \\
\hline Lepton reconstruction & $+2.7-2.6$ & $+2.1-1.9$ & $+1.7-1.6$ \\
Jet reconstruction $/ E_{\mathrm{T}}^{\text {miss }}$ & $+3.3-3.9$ & $+2.6-3.2$ & $+2.8-3.4$ \\
$b$-tagging & $+2.1-1.9$ & $+2.2-1.9$ & $+2.1-1.9$ \\
Backgrounds & $+2.8-3.0$ & $+1.8-2.1$ & $+1.7-2.1$ \\
\hline Monte Carlo generator & $-0.7+0.7$ & $+0.1-0.1$ & $-0.3+0.3$ \\
Parton shower/fragmentation & $+2.0-2.0$ & $+2.6-2.6$ & $+2.3-2.3$ \\
Initial-/final-state radiation & $-4.1+4.1$ & $-1.8+1.8$ & $-3.0+3.0$ \\
Parton distribution functions & $+6.2-6.0$ & $+5.6-5.9$ & $+5.9-5.9$ \\
\hline Total & $+9.4-9.5$ & $+7.9-8.2$ & $+8.2-8.4$ \\
\hline \hline Uncertainty on fiducial $\sigma_{t \bar{t}}$ & $e+$ jets & $\mu+$ jets & $\ell+$ jets \\
\hline Monte Carlo generator & $+1.5-1.5$ & $+1.8-1.8$ & $+1.6-1.6$ \\
Parton shower/fragmentation & $-2.6+2.6$ & $-3.1+3.1$ & $-2.9+2.9$ \\
Initial-/final-state radiation & $+0.4-0.4$ & $+0.2-0.2$ & $+0.3-0.3$ \\
\hline Total & $+9.1-8.8$ & $+8.0-8.5$ & $+7.9-8.3$
\end{tabular}

Transformed Aplanarity :

$$
A^{\prime}=\exp (-8 A)
$$

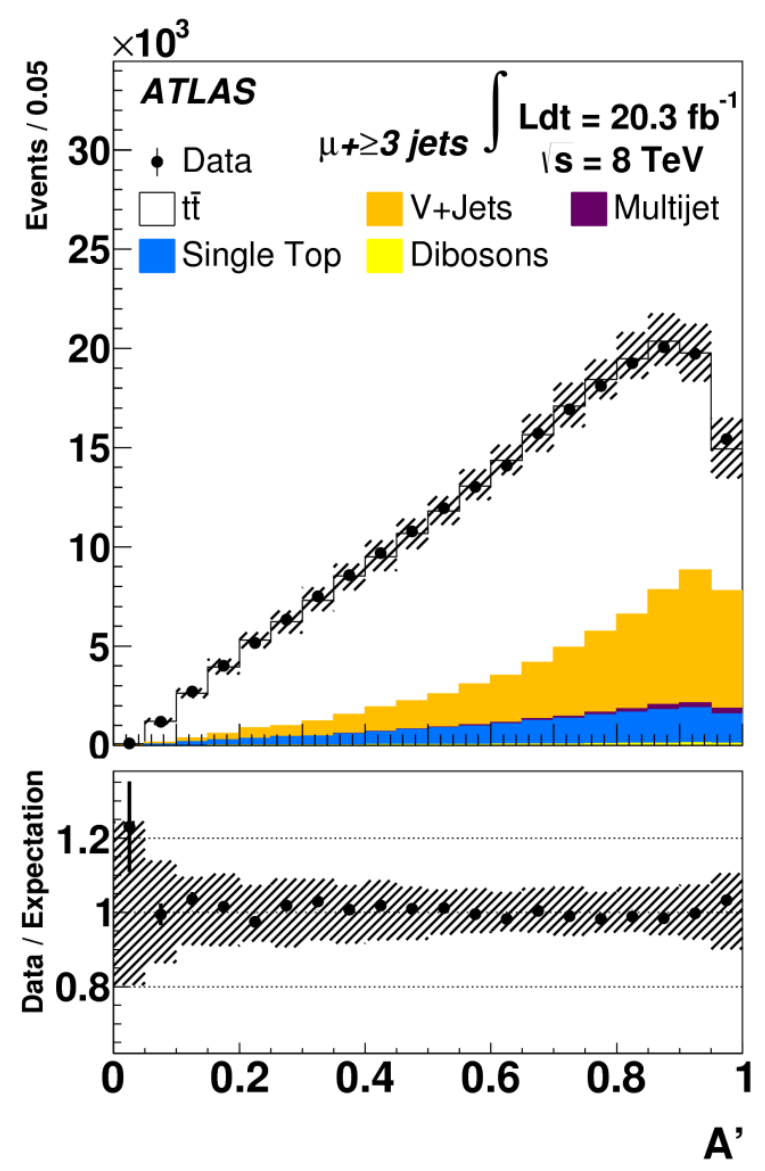




\section{Uncertainties between particle and parton level}

In the boosted top analysis, comparison of uncertainties

From detector to particle

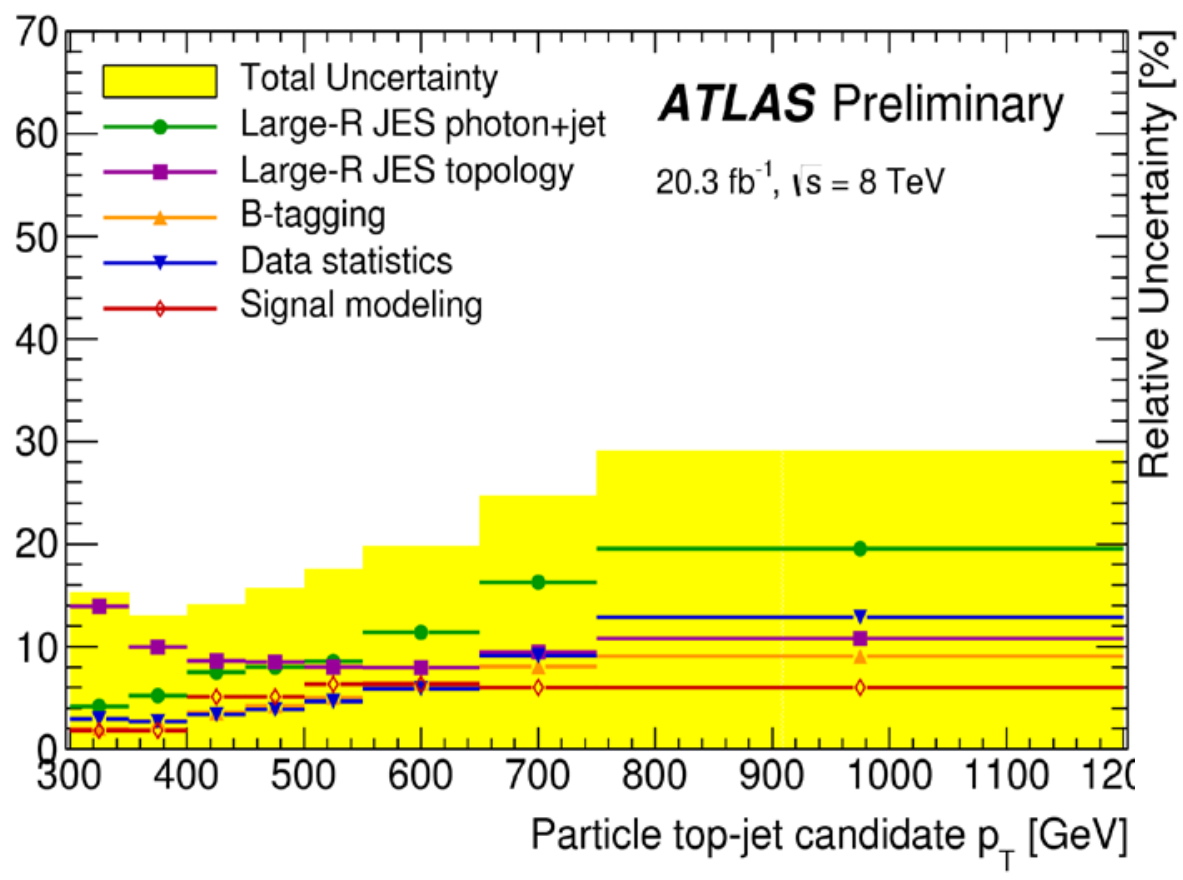

\section{From detector to parton}

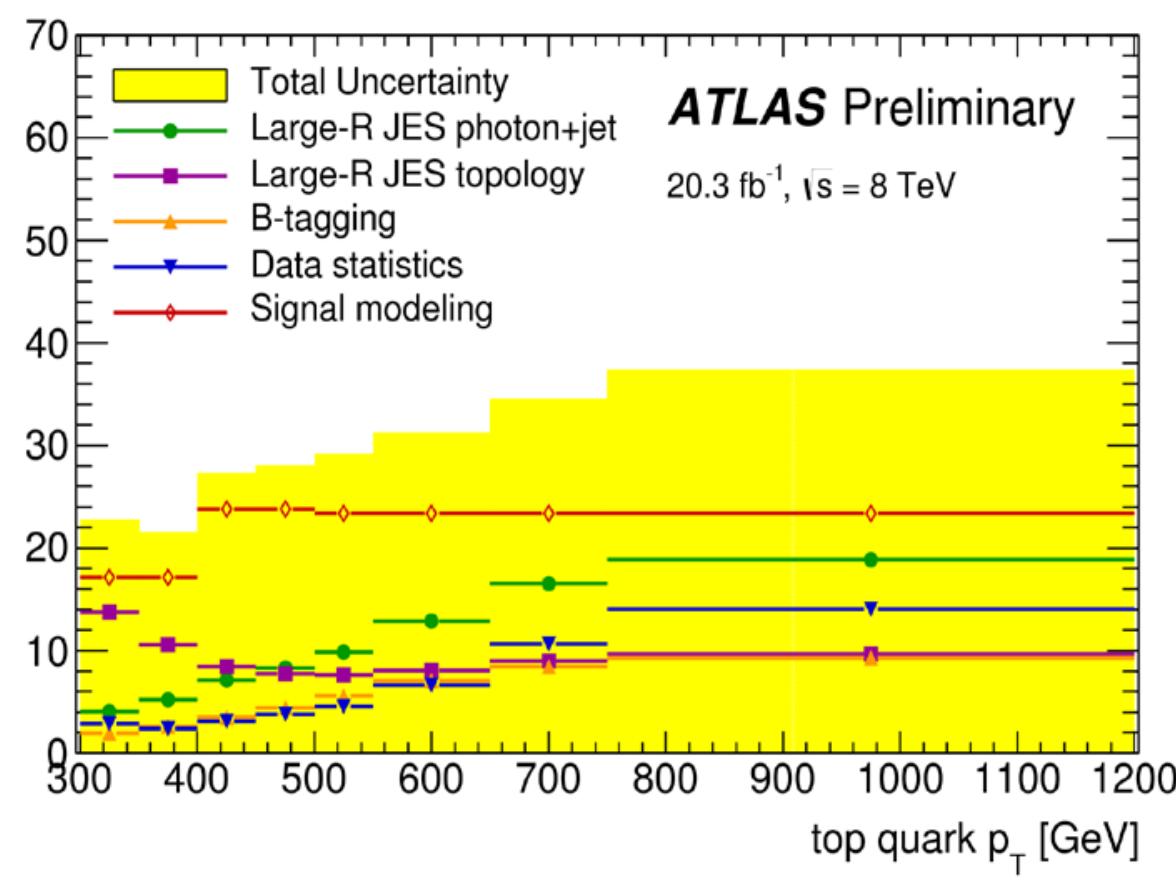




\section{Differential cross section for $t \bar{t}+j e t s$}

- The production of additional jets is sensitive to high-order perturbative QCD effect. The uncertainty associated is a source of uncertainties in other precision measurements.

- The cross section is also measured as function of each jet $P_{T}$

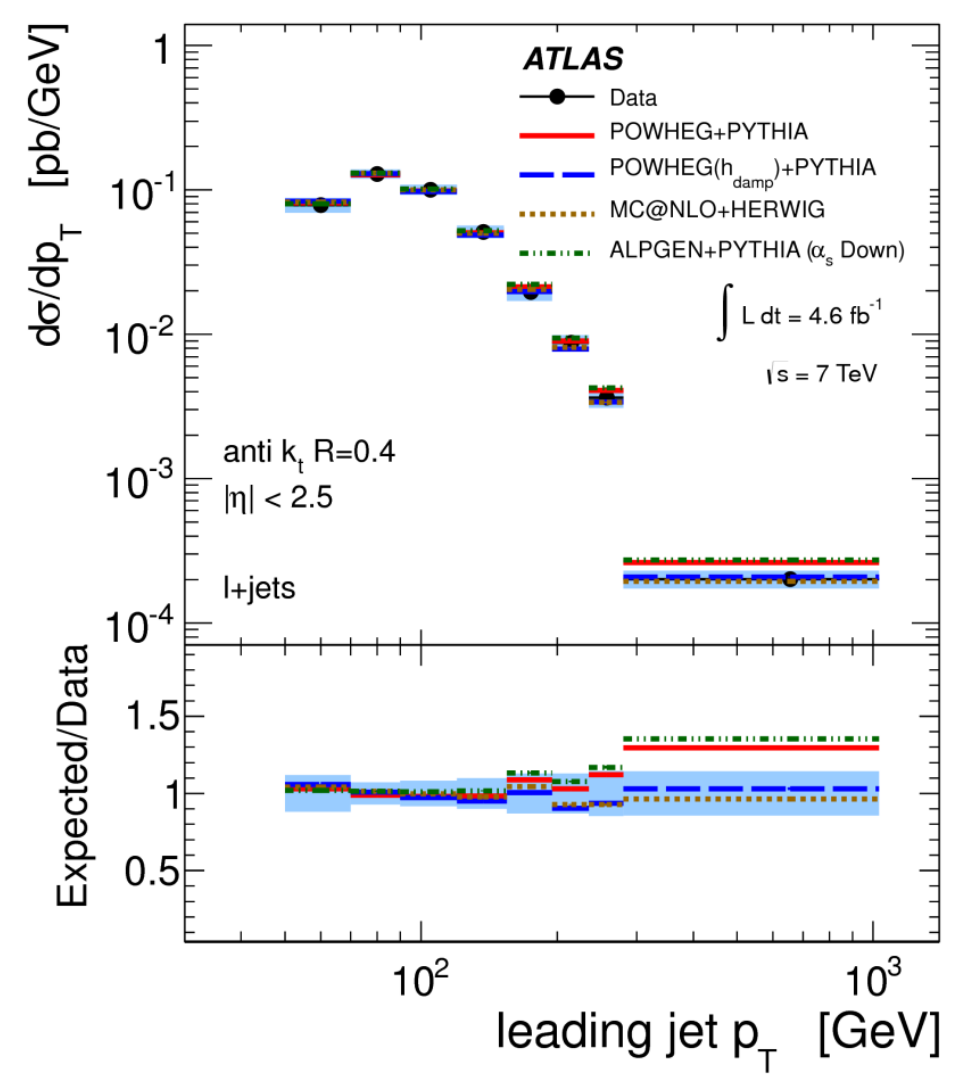




\section{$\overline{\mathrm{t}}+\gamma$ measurement}

Similar to $\ell+$ jets selection +1 tight photon (to suppress hadron decay like $\pi^{0} \rightarrow \gamma \gamma$ ) Cut on $\Delta R$ between lepton and jets.

Signal extraction with a template-based profile likelihood fit on the

$\mathrm{P}_{\mathrm{T}} \mathrm{iso}=\sum P_{T}$

tracks in a

cone $\Delta R=0.2$

around the photon

Backgrounds: Misidentified jets or electrons as photons and prompt photons.

$152 \pm 31$ signal events and $199 \pm 47$ background events

\begin{tabular}{c|c}
\hline \hline Uncertainty source & Uncertainty [\%] \\
\hline Background template shapes & 3.7 \\
Signal template shapes & 6.6 \\
\hline Signal modeling & 8.4 \\
\hline Photon modeling & 8.8 \\
Lepton modeling & 2.5 \\
Jet modeling & 16.6 \\
b-tagging & 8.2 \\
$E_{\mathrm{T}}^{\text {miss }}$ modeling & 0.9 \\
Luminosity & 1.8 \\
\hline Background contributions & 7.7 \\
\hline \hline
\end{tabular}




\section{$\mathrm{t} \overline{\mathrm{t}}+$ boson $(\mathrm{W}, \mathrm{Z})$ measurement}

\begin{tabular}{|c|c|c|c|c|c|}
\hline & \multicolumn{3}{|c|}{ Trilepton and same-sign dilepton } & \multicolumn{2}{|c|}{ Opposite-sign dilepton } \\
\hline Analysis strategy & \multicolumn{3}{|c|}{$\begin{array}{c}\text { comparable signal and background: } \\
\text { cut and count }\end{array}$} & \multicolumn{2}{|c|}{$\begin{array}{l}\text { small signal in huge background } \\
\text { multivariate techniques }\end{array}$} \\
\hline & $3 \ell \mathrm{Z}$ & $3 \ell Z$ veto & $2 \mu \mathrm{SS}$ & $2 \ell$ OSZveto & $2 \ell \mathrm{OSZ}$ \\
\hline Z-mass selection & $\left|m_{\ell \ell}-m_{Z}\right|<10 \mathrm{GeV}$ & $\left|m_{\ell \ell}-m_{Z}\right|>10 \mathrm{GeV}$ & - & $\left|m_{\ell \ell}-m_{Z}\right|>10 \mathrm{GeV}$ & $\left|m_{\ell \ell}-m_{Z}\right|<10 \mathrm{GeV}$ \\
\hline $\begin{array}{l}\text { Additional } \\
\text { selection }\end{array}$ & & & $\begin{array}{l}E_{\mathrm{T}}^{\text {miss }}>40 \mathrm{GeV} \\
H_{\mathrm{T}}>240 \mathrm{GeV}\end{array}$ & $\begin{array}{c}E_{\mathrm{T}}^{\text {miss }}>40 \mathrm{GeV}_{(e e, \mu \mu)} \\
H_{\mathrm{T}}>130 \mathrm{GeV}_{(e \mu)} \\
\Delta R_{\mathrm{ave}}^{\mathrm{ij}}>0.75\end{array}$ & $\Delta R_{\mathrm{ave}}^{\mathrm{ij}}>0.75$ \\
\hline $\begin{array}{l}\text { Lepton flavour } \\
\text { Signal } \\
\text { Main background }\end{array}$ & $\begin{array}{c}\text { all trilepton } \\
t \overline{t Z} \\
t Z, W Z \text { and fakes }\end{array}$ & $\begin{array}{c}\text { all trilepton } \\
t \bar{t} W \text { dominated } \\
t \bar{t} \mathrm{Z}, t \bar{t} H \text { and fakes }\end{array}$ & $\begin{array}{c}\mu \mu \\
t \bar{t} W \text { dominated } \\
t \bar{t} Z, t \bar{t} H \text { and fakes }\end{array}$ & $\begin{array}{l}\text { all dilepton } \\
t \overline{t Z} \text { and } t \bar{t} W \\
t \bar{t}+\text { jets }\end{array}$ & $\begin{array}{c}e e, \mu \mu \\
t \overline{t Z} \text { dominated } \\
Z+j e t s\end{array}$ \\
\hline Validation regions & $(3 \mathrm{j}+2 \mathrm{j}+1 \mathrm{j}, 1 \mathrm{~b})(\mathrm{CRZ})$ & (1b) (CRW) & $E_{\mathrm{T}}^{\text {miss }}<40 \mathrm{GeV}$ & & \\
\hline $\begin{array}{l}\text { Regions in the fit } \\
\text { (Signal region, } \\
\text { control region) }\end{array}$ & $\begin{array}{c}(\geq 4 \mathrm{j}, 1 \mathrm{~b})(\mathrm{SRB} 1 \mathrm{J4}) \\
(3 \mathrm{j}, \geq 2 \mathrm{~b})(\mathrm{SRB} 2 . J 3) \\
(\geq 4 \mathrm{j}, \geq 2 \mathrm{~b})(\mathrm{SRB} 2 . J 4)\end{array}$ & $(3 \mathrm{j}+2 \mathrm{j}, \geq 2 \mathrm{~b})(\mathrm{SRW} 3 \ell)$ & $(\geq 2 \mathrm{j}, \geq 2 \mathrm{~b})(\mathrm{SR} 2 \mu \mathrm{SS})$ & $\begin{array}{r}(3 \mathrm{j}, 1 \mathrm{~b}+2 \mathrm{~b}) \\
(4 \mathrm{j}, 1 \mathrm{~b}+2 \mathrm{~b}) \\
(\geq \mathbf{5 j}, 1 \mathrm{~b}+2 \mathrm{~b})\end{array}$ & $\begin{array}{r}(3 \mathrm{j}, 2 \mathrm{~b}) \\
(4 \mathrm{j}, 2 \mathrm{~b}) \\
(\geq \mathbf{5 j}, \mathbf{2 b})\end{array}$ \\
\hline
\end{tabular}

SM (NLO) predictions: $\sigma(\mathrm{ttZ})=206 \pm 29 \mathrm{fb}$ and $\sigma(\mathrm{ttW})=203 \pm 25 \mathrm{fb}$

Measured : $\sigma(\mathrm{ttZ})=150_{-50}^{+55}$ (stat) \pm 21 (syst) fb

$$
\sigma(\mathrm{ttW})=300_{-100}^{+120} \text { (stat) }{ }_{-40}^{+70} \text { (syst) fb }
$$

For each, $3.1 \sigma$ excess over the bkg-only hypothesis 